\title{
Differential transcript expression between the microfilariae of the filarial nematodes, Brugia malayi and B. pahangi
}

\author{
Michael M Kariuki', Leonard B Hearne² and Brenda T Beerntsen*1
}

\begin{abstract}
Background: Brugia malayi and B. pahangi are two closely related nematodes that cause filariasis in humans and animals. However, B. pahangi microfilariae are able to develop in and be transmitted by the mosquito, Armigeres subalbatus, whereas most B. malayi are rapidly melanized and destroyed within the mosquito hemocoel. A crossspecies microarray analysis employing the $B$. malayi $V 2$ array was carried out to determine the transcriptional differences between B. malayi and B. pahangi microfilariae with similar age distribution.
\end{abstract}

Results: Following microarray data analysis, a list of preferentially expressed genes in both microfilariae species was generated with a false discovery rate estimate of $5 \%$ and a signal intensity ratio of 2 or higher in either species. A total of 308 probes were preferentially expressed in both species with 149 probes, representing 123 genes, in B. pahangi microfilariae and 159 probes, representing 107 genes, in B. malayi microfilariae. In B. pahangi, there were 76 (62\%) upregulated transcripts that coded for known proteins that mapped into the KEGG pathway compared to 61 (57\%) transcripts in B. malayi microfilariae. The remaining 47 (38\%) transcripts in B. pahangi and 46 (43\%) transcripts in B. malayi microfilariae were comprised almost entirely of hypothetical genes of unknown function. Twenty-seven of the transcripts in B. pahangi microfilariae coded for proteins that associate with the secretory pathway compared to thirtynine in B. malayi microfilariae. The data obtained from real-time PCR analysis of ten genes selected from the microarray list of preferentially expressed genes showed good concordance with the microarray data, indicating that the microarray data were reproducible.

Conclusion: In this study, we identified gene transcripts that were preferentially expressed in the microfilariae of $B$. pahangi and B. malayi, some of which coded for known immunomodulatory proteins. These comparative transcriptome data will be of interest to researchers keen on understanding the inherent differences, at the molecular level, between B. malayi and B. pahangi microfilariae especially because these microfilariae are capable of surviving in the same vertebrate host but elicit different immune response outcomes in the mosquito, Ar. subalbatus.

\section{Background}

Lymphatic filariasis is caused by the thread-like parasitic nematodes Wuchereria bancrofti, Brugia malayi and B. timori which are transmitted by mosquitoes. B. malayi and $B$. timori are the main cause of Brugian lymphatic filariasis in humans and B. pahangi causes filariasis in domestic cats, dogs and wild animals. The periodic form of $B$. malayi primarily infects humans whereas the subperiodic form is zoonotic [1]. Both B. malayi and B. pah-

*Correspondence: beerntsenb@missouri.edu

1 Department of Veterinary Pathobiology, College of Veterinary Medicine, University of Missouri, Columbia, MO 65211, USA

Full list of author information is available at the end of the article angi have similar life cycles in their natural vertebrate and mosquito hosts. The adult worms of both species reside in the lymphatic vessels of infected vertebrates and, when mature, produce sheathed microfilariae that enter the blood stream. When a competent mosquito acquires microfilariae in a blood meal, the microfilariae develop within the mosquito into third stage larval forms that are infective to vertebrates.

The B. malayi and B. pahangi microfilariae are morphologically similar, but genetically these worms can be differentiated by restriction site polymorphism within specific regions of repeated DNA sequences [2-4]. Physiologically, both B. malayi and B. pahangi microfilariae 
are able to develop to infective stage larvae in Anopheles quadrimaculatus and Aedes aegypti [5]. However, only $B$. pahangi microfilariae are able to develop in and be transmitted to the vertebrate host by the natural vector, Armigeres subalbatus, whereas most of the $B$. malayi microfilariae are rapidly and effectively destroyed in the hemocoel by melanotic encapsulation [6-8]. Melanin synthesis in mosquitoes involves a series of complex biochemical reactions that are triggered in response to either injury to the insect body or by molecules on the surface of invading microbial pathogens [9].

The antigens on the surface of $B$. malayi and B. pahang $i$ microfilariae have been found to be similar, with antibodies raised against surface antigens of one species crossreacting with surface antigens from the other species [10]. However, the protein profile of microfilaria surface antigens seen on SDS-PAGE analysis shows differences in molecular weight of some surface antigens, indicating that there are species-specific molecules on the $B$. malayi and $B$. pahangi microfilariae surfaces [10]. The two outer layers of the microfilariae, the sheath and cuticle, play an important role in host-parasite interactions and have been shown to contain carbohydrate epitopes on their surfaces $[11,12]$. Immunochemical studies have shown that during B. malayi microfilariae maturation, the composition of the surface antigens changes and results in differences in mosquito infectivity between immature and mature microfilariae [13]. With regard to mosquito immunity, carbohydrate molecules and other pathogenassociated molecules located on the surface on invading pathogens are recognized by mosquito pattern recognition receptors in a species-specific manner and lead to the activation of mosquito defenses such as phagocytosis and melanotic encapsulation [14]. It is therefore possible that subtle differences in the carbohydrate or protein content of the B. malayi and B. pahangi microfilariae surface may be responsible for the species-specific melanotic encapsulation response of $A r$. subalbatus towards $B$. malayi and $B$. pahangi microfilariae. It is also a possibility that $B$. pahangi microfilariae secrete molecules that modulate the melanotic encapsulation response mounted by Ar. subalbatus.

During the past decade, microarray analysis has paved the way for thorough studies of gene expression $[15,16]$. More recently, cross-species hybridization analysis has been used as a way to investigate differences between two closely related species [17-19]. In this study, we sought to determine the differences between $B$. pahangi and subperiodic $B$. malayi microfilariae at the transcriptional level using cross-species microarray analysis. First, we identified the global molecular differences that exist between the transcriptomes of the microfilariae of these two species. Second, we identified transcripts that are preferentially expressed in one species and whose products are likely to be secreted or located on the surface of the microfilariae. Proteins that are secreted by microfilariae or located on their surface are likely to play a significant role in the interaction of microfilariae with their vertebrate and mosquito hosts.

\section{Results}

Sequence comparison between B. pahangi and B. malayi genes

We selected twenty-two nucleotide sequences of B. pahangi genes, complete or partial, deposited in GenBank and compared their nucleotide sequences to their counterpart genes in B. malayi (Table 1). The alignment results showed that, on average, $B$. malay $i$ and $B$. pahangi genes had $97 \%$ nucleotide sequence identity. During cross-species microarray hybridizations, a sequence mismatch between the probe and the target sequence in one species can lead to inaccurate interpretations of target levels between the species. In this study, we sought to determine whether there were sequence differences between $B$. malayi and $B$. pahangi genes particularly in the sequence used as probes and spotted on the array. To this end, we randomly selected five $B$. malayi genes whose probes were present on the $\mathrm{BmV} 2$ array and designed primers that flanked the probe sequence. We carried out PCRs using these primers on B. malay $i$ and $B$. pahangi genomic DNA templates and sequenced the PCR products. The alignment results showed that the oligonucleotide sequence of the selected probes were a match for both the B. malayi and B. pahangi genes (Figure 1). In one case, the bm.02018 probe derived from Pub Locus Bm1_34045 appears to straddle an exon-intron junction as evidenced by the consensus dinucleotides found at each end of the intron, GT at the $5^{\prime}$ end and AG at the 3 ' end of the intron [20].

\section{Transcript expression profiles of B. pahangi and B. malayi microfilariae genes}

A draft of the whole $B$. malayi genome was recently published [21] and of the $15412 \mathrm{~B}$. malayi probes on the BmV2 array, 11975 probes had matching sequences. Of these 11975 probes, statistical analysis of the microarray data revealed that a total 877 probes (7\%) had signal intensities above threshold in at least one species with 504 probes higher in B. pahangi and 373 probes higher in B. malayi. Probes with FDR estimates of $5 \%$ and signal intensity ratios of 2 or higher, with respect to either species, were considered preferentially expressed. There were 149 probes that had signal intensity ratios of 2 or higher in B. pahangi microfilariae and 159 probes in $B$. malayi microfilariae. The 149 probes identified in B. pahangi represented 123 genes and the 159 probes identified 


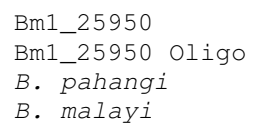

----AGGAAACTGGATTCAATTCTCCAAGTTTCATATTTCCGTACAGTACTGTGTTCAATGCTAATCAT--TACGAAGGAAACTGGATTCAATTCTCCAAGTTTCATATTTCCGTACAGTACTGTGTTCAATGCTAATCATCCA TACGAAGGAAACTGGATTCAATTCTCCAAGTTTCATATTTCCGTACAGTACTGTGTTCAATGCTAATCATCCA

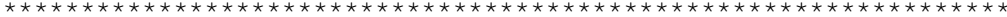

-----TTATTTCATGTGCCCTGCAATGTTGAGAAAATACTAAAGGCGGATTACGGTAATTGGCGAGTACC--GAAGGTTATTTCATGTGCCCTGCAATGTTGAGAAAATACTAAAGGCGGATTACGGTAATTGGCGAGTACCTCA GAAGGTTATTTCATGTGCCCTGCAATGTTGAGAAAATACTAAAGGCGGATTACGGTAATTGGCGAGTACCTCA

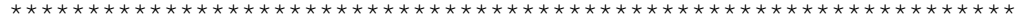

---AAGAAGATATTTTGGTACTAACTGGACTTATTCGCGCTGATAGCAATGTTTCGGATAATAGTGAT--CGAAAAAGAAGATATTTTGGTACTAACTGGACTTATTCGCGCTGATAGCAATGTTTCGGATAATAGTGATGAT CGAAGAAGAAGATATTTTGGTACTAACTGGACTTATTCGCGCTGATAGCAATGTTTCGGATAATAGTGATGAT

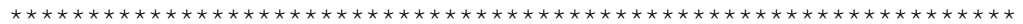

-----ACTCAAGATGTTATCTTATTCGGTCGATTCTATCAATCCACTAATAGTTTTAATGGAGCGGCCTA--CACTTACTCAAGATGTTATCTTATTCGGTCGATTCTATCAATCCACTAATAGTTTTAATGGAGCGGCCTATTA CACTTACTCAAGATGTTATCTTATTCGGTCGATTCTATCAATCCACTAATAGTTTTAATGGAGCGgTATGTTA

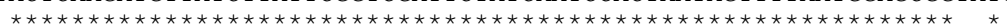

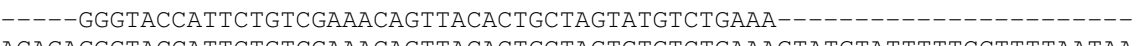
ACAGAGGGTACCATTCTGTCGAAACAGTTACACTGCTAGTGTGTCTGAAAGTATGTATTTTTCCTTTTAATAA ACAGAGGGTACCATTCTGTCGAAACAGTTACACTGCTAGTATGTCTGAAAGTATGTATTTTTCCTTTTAATAA

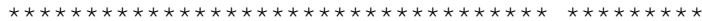

CGGTTATAATTTCATTAAATTTTCTGTGATCTGAAT CGGTTATAATTTCATTAAATTTTCTGTGATCTGAATTGAATTTTATTTTATTGAATTCTCTGTGATTTCAATG TGTTTCAGGTTCGCTATAAAGAACGTGTTACCATTCTGCGAGGCAATCATGAGTCACGGCAGATCACTCAGGT

Figure 1 Sequence alignment of five probes against the PCR products of $\boldsymbol{B}$. pahangi and $\boldsymbol{B}$. malayi. Primers designed to flank the probe sequence of five probes on the BmV2 chip that had three to ten times higher signal intensities in B. malayi microfilariae than in B. pahangi were used for PCRs with B. pahangi and B. malayi genomic DNA as templates and the PCR product was sequenced. The asterisks indicate identical nucleotides in the aligned sequences. For Bm1_34045, the consensus dinucleotides at either ends of the intron, GT at the 5' end and AG at the 3' end, are shown in bold for both the B. pahangi and B. malayi genes.

in B. malayi represented 107 genes (Table 2) as some genes were represented by more than one probe on the BmV2 array.

\section{Functional classification of preferentially expressed gene transcripts}

Not all the genes represented on the $\mathrm{BmV} 2$ array had Gene Ontology (GO) assignments. Of the 123 gene transcripts found to be preferentially up-regulated in B. pahangi microfilariae, 76 (62\%) of them coded for known proteins that mapped into KEGG pathways and the remaining 47 (38\%) transcripts encoded mostly hypothetical proteins of unknown function. Of the 76 known genes, there were 14 genes involved in cellular processes, 9 genes in environmental information processes, 37 genes in genetic information processing and 16 genes in metabolism (Additional File 1).
Of the 107 gene transcripts preferentially up-regulated in B. malayi microfilariae, 61 (57\%) of them coded for known proteins that mapped into KEGG pathways and the remaining 46 (43\%) transcripts encoded mostly hypothetical proteins of unknown function. Of the 61 known genes, there were 9 genes involved in cellular processes, 6 genes in environmental information processes, 38 genes in genetic information processing and 8 genes in metabolism (Additional File 2). A comparison of the preferential expressed gene transcripts in both $B$. pahangi and $B$. malayi based upon their overall KEGG pathway association is shown in Figure 2.

\section{Predicted localization of proteins encoded by preferentially expressed gene transcripts}

The proteins coded for by the transcripts found to be preferentially up-regulated in B. malayi and B. pahangi 
Table 1: Nucleotide sequence similarity between B. pahangi and B. malayi genes.

\begin{tabular}{|c|c|c|c|c|c|}
\hline Accession \# & Brugia Gene & Identical & Total & $\%$ Identity & Gaps \\
\hline$\underline{\text { AF013991 }}$ & TGF-b type I receptor & 1912 & 1942 & 98 & 2 \\
\hline$\underline{U} 45314$ & Microfilarial sheath protein & 1883 & 2007 & 93 & 55 \\
\hline AJ005784 & Heat shock protein 90 & 1796 & 1822 & 98 & 6 \\
\hline$\underline{A A S 18673}$ & Gamma-glutamyl transpeptidase precusor & 1756 & 1773 & 99 & 0 \\
\hline$\underline{X 69128}$ & Glutathione peroxidase & 1504 & 1582 & 98 & 45 \\
\hline$\underline{M 27191}$ & Heat shock protein 70 (hsp70) mRNA & 1202 & 1228 & 97 & 7 \\
\hline AF031819 & Cathepsin L-like cysteine protease & 1133 & 1188 & 95 & 10 \\
\hline$\underline{\text { U59690 }}$ & Chitinase partial CDs & 1126 & 1153 & 97 & 8 \\
\hline AJ249374 & Brugia pahangi col-1 gene cuticular collagen & 864 & 870 & 99 & 0 \\
\hline$\underline{A J 130821}$ & Alt-1 protein & 617 & 636 & 97 & 3 \\
\hline$\underline{\mathrm{AJ} 271611}$ & Cytochrome oxidase subunit I partial CDs & 573 & 632 & 90 & 6 \\
\hline$\underline{X 76283}$ & Extracellular Cu/Zn-superoxide dismutase & 550 & 584 & 94 & 17 \\
\hline$\underline{X 87901}$ & Small heat shock protein & 525 & 534 & 98 & 0 \\
\hline$\underline{X 76284}$ & Cytosolic Cu/Zn-superoxide dismutase & 483 & 493 & 97 & 2 \\
\hline AY050257 & $\begin{array}{l}\text { Fatty acid retinoid binding protein precursor } \\
(\mathrm{far}-1) \mathrm{mRNA}\end{array}$ & 474 & 477 & 99 & 0 \\
\hline$\underline{X 91065}$ & Cytidine deaminase & 399 & 402 & 99 & 0 \\
\hline EF413918 & Myosin partial CDs & 384 & 390 & 98 & 1 \\
\hline$\underline{X 95663}$ & Elongation factor 1 alpha partial CDs & 365 & 375 & 97 & 1 \\
\hline \multirow[t]{2}{*}{$\underline{\mathrm{AJ} 224966}$} & Brugia pahangi mRNA for tropomyosin & 170 & 171 & 99 & 0 \\
\hline & Total & 19070 & 19630 & $($ Avg) 97 & \\
\hline
\end{tabular}

The partial or complete nucleotide sequences of twenty-two B. pahangi genes deposited in GenBank were run on the NCBI's nucleotide BLAST program. The output of the BLAST analysis assigned to the corresponding B. malayi gene is shown above.

were grouped according to whether they were secretory or non-secretory as indicated by the SignalP [22] predictions provided on the BmV2 array annotation. Of the 123 genes up-regulated in $B$. pahangi microfilariae, 27 genes (22\%) coded for proteins containing a signal sequence on the $\mathrm{N}$-terminus implying that these proteins associate with the secretory pathway while the remaining 96 genes (78\%) coded for proteins without a signal sequence. Of the 27 proteins associated with the secretory pathway, TargetP [23] predictions indicate that 3 proteins are likely to be located in the mitochondria, 16 proteins are likely

Table 2: Overall number of preferentially expressed transcripts in B. pahangi and B. malayi microfilariae.

\begin{tabular}{|c|c|c|c|}
\hline $\begin{array}{l}\text { Microarray data treatment at 5\% FDR } \\
\text { estimate }\end{array}$ & $\mathrm{Bp} / \mathrm{Bm}$ & $\mathrm{Bm} / \mathrm{Bp}$ & Total \\
\hline Probe signal intensity ratio $>=1$ & $504(57 \%)$ & $373(43 \%)$ & 877 \\
\hline Probe signal intensity ratio $>=2$ & $149(48 \%)$ & $159(52 \%)$ & 308 \\
\hline a Genes with signal intensity ratio $>=2$ & $123(53 \%)$ & 107 (47\%) & 230 \\
\hline
\end{tabular}




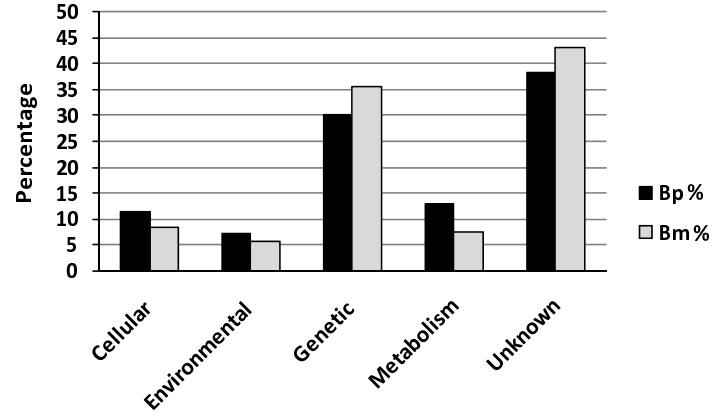

Figure 2 KEGG pathway classification of preferentially expressed genes in B. pahangi and B. malayi microfilariae. The genes that were classified as up-regulated in either species were manually mapped into the KEGG pathway groups. Cellular; Cellular Processes, Environmental; Environmental Information Processing, Genetic; Genetic Information Processing.

to be secreted and 8 have an unknown destination (Table 3).

Similarly, in B. malayi, SignalP predictions on the $\mathrm{BmV} 2$ array annotation suggested that, of the 107 genes up-regulated in B. malayi microfilariae, 39 genes (36\%) coded for proteins that associate with the secretory pathway and the remaining 68 genes (64\%) were denoted as non-secretory because of the lack of a signal sequence on the $\mathrm{N}$-terminus of the protein. Of the 39 proteins associated with the secretory pathway, TargetP predictions suggested that 2 proteins were likely to be localized in the mitochondria, 32 proteins were likely to be secreted and 5 had an unknown destination (Table 4).

The 27 B. pahangi and 39 B. malayi predicted secretory proteins coded for by preferentially expressed transcripts were further analyzed by PSORTII [24] and ProtComp http://www.softberry.com; two protein algorithm that give more detailed protein localization predictions. Of the $27 \mathrm{~B}$. pahangi proteins, 20 proteins were predicted to be either extracellular or on the plasma membrane by PSORTII or ProtComp with the remaining 7 proteins being predicted to be in the cytoplasm, Golgi apparatus, endoplasmic reticulum, peroxisome, mitochondria or nucleus (Table 3). Of the 39 B. malayi proteins with signal sequences, 28 proteins were predicted to be either extracellular or on the plasma membrane by PSORTII or ProtComp with the remaining 11 proteins suggested as being localized in the cytoplasm, Golgi apparatus, endoplasmic reticulum, peroxisome, mitochondria or nucleus (Table 4).

\section{Validation of microarray data by real-time PCR analysis}

To verify the microarray data, 13 Brugia genes were selected for real-time PCR analysis (Table 5). The genes were selected based on the signal intensities of their probes, in either species, from the microarray data. Five of the selected genes had higher signal intensities in $B$. pahangi, five in B. malayi and the remaining three had similar signal intensities in both species. First, it was important to identify a gene with equal transcript levels in both species that was to be use as an endogenous reference. To this end, primers were designed that amplified between 200-300 base pair fragments of the Pub Locus represented by the three probes, bm.02018, BMX9555 and BMX10185, that showed similar signal intensities in the microarray data. Real-time PCR analysis was performed using these primers on CDNA derived from five biological samples of B. pahangi and B. malayi microfilariae with similar age distribution. Of the three probes with similar signal intensities in both species on the microarray data, the bm.02018 probe, which was derived from the Pub Locus Bm1_34045, was found to have a Ct ratio closest to the value one, across all five biological samples (Figure 3A). For this reason, Bm1_34045 was assigned as the endogenous reference to which the remaining ten genes were normalized. The comparative $\mathrm{Ct}$ method was used to determine preferentially expressed transcripts [25]. The Brugia microfilaria samples used for real-time PCR were independent of those used in the microarray analysis. The comparison of realtime PCR data to that obtained in the microarray analysis showed the same trend and a strong correlation between the data obtained by the two methods using different biological samples (Figure 3B), suggesting that the transcriptional profiles derived from the microarray data are accurate and reproducible.

\section{Discussion}

This study was a cross-species comparison of gene transcription between the microfilariae of $B$. malay $i$ and $B$. pahangi. The B. pahangi and B. malayi microfilariae used in this study were both obtained from similar micro-environments; the intra-peritoneal cavity of experimentallyinfected jirds. Therefore, it would be safe to assume that the difference in the transcriptional profile of each nematode species was intrinsic to each species and not due to micro-environmental influence.

In order to absorb any possible variation in microfilaria gene transcription between the two species due to the host immune responses or slight differences in microfilaria age distribution, independent biological samples of B. malayi and B. pahangi microfilariae were collected from eight pairs of jirds and used in this study. Three paired biological samples were used for the microarray analysis and five paired biological samples, different from those used for microarray analysis, were used in the real time PCR analysis to confirm the validity of the microarray data. For the microarray analysis, there were 3 technical replicates for each of the 3 biological samples, 
Table 3: Localization predictions for proteins encoded by preferentially expressed transcripts in B. pahangi microfilariae.

\begin{tabular}{|c|c|c|c|c|c|}
\hline Pub Locus & Annotation & SignalP & TargetPa & PSORTII & ProtComp \\
\hline Bm1_49810 & Conserved hypothetical protein & Signal & mitochondria (5) & $\mathrm{CY}$ & PM \\
\hline Bm1_44755 & RE21922p, putative & Signal & mitochondria (5) & ER & PM \\
\hline Bm1_23875 & Hypothetical protein & Signal & mitochondria (5) & $\mathrm{GO}$ & $\mathrm{ER}$ \\
\hline Bm1_03995 & Serpin (BmSERPIN), putative & Signal & secreted (1) & ER & ER \\
\hline Bm1_02070 & Serpin, putative & Signal & secreted (1) & ER & $\mathrm{ER}$ \\
\hline Bm1_32890 & Histidine acid phosphatase family protein & Signal & secreted (1) & ER & MT \\
\hline Bm1_07905 & Metallophosphoesterase 1, putative & Signal & secreted (1) & ER & PM \\
\hline Bm1_42865 & Brugia malayi antigen, putative & Signal & secreted (1) & EX & EX \\
\hline Bm1_40635 & Conserved hypothetical protein & Signal & secreted (1) & EX & EX \\
\hline Bm1_37310 & $\begin{array}{l}\text { FKBP-type peptidyl-prolyl cis-trans isomerase- } \\
\text { 13, BmFKBP-13 }\end{array}$ & Signal & secreted (1) & EX & EX \\
\hline Bm1_47735 & Lipase family protein & Signal & secreted (1) & EX & EX \\
\hline Bm1_49635 & Protein $\mathrm{C} 17 \mathrm{H} 12.11$, putative & Signal & secreted (1) & EX & EX \\
\hline Bm1_53990 & Zinc finger, $\mathrm{C} 2 \mathrm{H} 2$ type family protein & Signal & secreted (1) & EX & NU \\
\hline Bm1_39915 & Transthyretin-like family protein & Signal & secreted (2) & $\mathrm{CY}$ & EX \\
\hline Bm1_00735 & Hypothetical protein & Signal & secreted (2) & $\mathrm{CY}$ & NU \\
\hline Bm1_50605 & Ribonuclease T2 family protein & Signal & secreted (2) & ER & EX \\
\hline Bm1_28420 & DnaJ protein, putative & Signal & secreted (2) & EX & EX \\
\hline Bm1_56195 & $\begin{array}{l}\text { Transforming growth factor b homolog, } \\
\text { putative }\end{array}$ & Signal & secreted (2) & EX & EX \\
\hline Bm1_33495 & $\begin{array}{l}\text { Probable protein disulfide isomerase A6 } \\
\text { precursor, putative }\end{array}$ & Signal & secreted (3) & EX & ER \\
\hline Bm1_25940 & Serpin, putative & Signal & unknown (2) & ER & EX \\
\hline Bm1_35905 & Hypothetical protein & Signal & unknown (3) & ER & PM \\
\hline Bm1_43565 & MBOAT family protein & Signal & unknown (3) & PM & ER \\
\hline Bm1_18845 & GRIM-19 protein & Signal & unknown (4) & $\mathrm{CY}$ & $\mathrm{GO}$ \\
\hline Bm1_37335 & Ankyrin-related unc-44, putative & Signal & unknown (4) & NU & EX \\
\hline Bm1_40630 & Hypothetical protein & Signal & unknown (5) & $\mathrm{CY}$ & PM \\
\hline Bm1_24145 & ABC1 family protein & Signal & unknown (5) & ER & PE \\
\hline Bm1_29610 & Hypothetical protein & Signal & unknown (5) & EX & NU \\
\hline
\end{tabular}

a Values in parentheses from TargetP results represent the reliability class (RC), from 1 to 5, where 1 indicates the strongest prediction. For PSORTII, location with the highest percentage score is shown. For ProtComp, the integral prediction of protein location is shown. CY, cytoplasm; ER, endoplasmic reticulum; EX, extracellular; GO, Golgi apparatus; NU, nuclear; PE, peroxisome; PM, plasma membrane; MT, mitochondria.

resulting in the use of nine microarray slides. Only those genes with similar signal intensity values across all nine microarrays, and by extension all 3 biological samples, were further analyzed and are shown on the gene lists. As a result, any influence on microfilaria gene transcription arising from subtle differences in microfilaria age distribution between the two species, immune pressure on the microfilaria by the vertebrate immune response, or artifacts of sample preparation was therefore greatly reduced.

The BmV2 array used in this study consisted of probes designed from $B$. malayi genomic gene models and ESTs.
Since the probes were an exact match for B. malayi genes and not necessarily proven to be so for the corresponding B. pahangi genes, one could assume that hybridizations on the BmV2 array would be biased towards B. malayi. Sequence divergence in some genes, particularly a sequence mismatch within the oligonucleotides used as a probe, could influence the hybridization kinetics and result in misleading data with respect to a cross-species differential gene transcription comparison [26,27].

In this study, we found that the $B$. pahangi genes with sequences deposited in GenBank had a high sequence identity to their counterpart B. malayi genes. In addition, 
Table 4: Localization predictions of proteins encoded by preferentially expressed transcripts in B. malayi microfilariae.

\begin{tabular}{|c|c|c|c|c|c|}
\hline Pub Locus & Annotation & SignalP & TargetPa & PSORTII & ProtComp \\
\hline Bm1_12070 & $\mathrm{K}+$ channel tetramerisation domain containing protein & Signal & mitochondria (5) & GO & PM \\
\hline Bm1_02255 & Zinc finger, $\mathrm{C} 2 \mathrm{H} 2$ type family protein & Signal & mitochondria (5) & NU & NU \\
\hline Bm1_14030 & Hypothetical protein & Signal & secreted (1) & $\mathrm{CY}$ & EX \\
\hline Bm1_56305 & Leucyl aminopeptidase, putative & Signal & secreted (1) & ER & EX \\
\hline Bm1_50670 & Potassium channel chain n2P18 homolog, putative & Signal & secreted (1) & $\mathrm{ER}$ & EX \\
\hline Bm1_30525 & Hypothetical protein & Signal & secreted (1) & EX & EX \\
\hline Bm1_33955 & Hypothetical protein & Signal & secreted (1) & EX & EX \\
\hline Bm1_19100 & Major microfilarial sheath protein precursor, putative & Signal & secreted (1) & EX & EX \\
\hline Bm1_10870 & Zinc finger, $\mathrm{C} 2 \mathrm{H} 2$ type family protein & Signal & secreted (1) & $\mathrm{NU}$ & $\mathrm{CY}$ \\
\hline Bm1_09090 & Zinc finger, $\mathrm{C} 2 \mathrm{H} 2$ type family protein & Signal & secreted (1) & NU & ER \\
\hline Bm1_55930 & Nematode cuticle collagen $\mathrm{N}$-terminal domain containing protein & Signal & secreted (1) & NU & EX \\
\hline Bm1_18365 & Zinc finger, $\mathrm{C} 2 \mathrm{H} 2$ type family protein & Signal & secreted (1) & NU & EX \\
\hline Bm1_22980 & Zinc finger, $\mathrm{C} 2 \mathrm{H} 2$ type family protein & Signal & secreted (1) & NU & EX \\
\hline Bm1_35265 & Zinc finger, $\mathrm{C} 2 \mathrm{H} 2$ type family protein & Signal & secreted (1) & NU & EX \\
\hline Bm1_34385 & Hypothetical protein & Signal & secreted (1) & NU & GO \\
\hline Bm1_04225 & Zinc finger, $\mathrm{C} 2 \mathrm{H} 2$ type family protein & Signal & secreted (1) & $\mathrm{NU}$ & GO \\
\hline Bm1_11185 & Zinc finger, $\mathrm{C} 2 \mathrm{H} 2$ type family protein & Signal & secreted (1) & NU & GO \\
\hline Bm1_13985 & Zinc finger, $\mathrm{C} 2 \mathrm{H} 2$ type family protein & Signal & secreted (1) & $\mathrm{NU}$ & GO \\
\hline Bm1_47425 & Zinc finger, $\mathrm{C} 2 \mathrm{H} 2$ type family protein & Signal & secreted (1) & $\mathrm{NU}$ & $\mathrm{GO}$ \\
\hline Bm1_21005 & Hypothetical protein & Signal & secreted (1) & NU & MT \\
\hline Bm1_22820 & Hypothetical protein & Signal & secreted (2) & $\mathrm{CY}$ & EX \\
\hline Bm1_45345 & Hypothetical 31.4 kDa protein T19C3.2 chromosome III, putative & Signal & secreted (2) & ER & EX \\
\hline Bm1_22110 & Hypothetical protein & Signal & secreted (2) & ER & PM \\
\hline Bm1_33830 & Prismalin-14, putative & Signal & secreted (2) & MT & EX \\
\hline Bm1_23300 & Zinc finger, $\mathrm{C} 2 \mathrm{H} 2$ type family protein & Signal & secreted (2) & NU & EX \\
\hline Bm1_03355 & Hypothetical protein & Signal & secreted (3) & $\mathrm{CY}$ & EX \\
\hline Bm1_08145 & Zinc finger, $\mathrm{C} 2 \mathrm{H} 2$ type family protein & Signal & secreted (3) & NU & NU \\
\hline Bm1_44495 & Hypothetical protein & Signal & secreted (4) & $\mathrm{CY}$ & EX \\
\hline Bm1_00295 & Hypothetical protein & Signal & secreted (4) & ER & PM \\
\hline Bm1_47450 & Conserved hypothetical protein, putative & Signal & secreted (4) & GO & EX \\
\hline Bm1_31055 & RNA recognition motif containing protein, putative & Signal & secreted (4) & NU & EX \\
\hline Bm1_15340 & Zinc finger, $\mathrm{C} 2 \mathrm{H} 2$ type family protein & Signal & secreted (4) & NU & NU \\
\hline Bm1_03710 & Zinc finger, $\mathrm{C} 2 \mathrm{H} 2$ type family protein & Signal & secreted (5) & $\mathrm{NU}$ & EX \\
\hline Bm1_05155 & Zinc finger, $\mathrm{C} 2 \mathrm{H} 2$ type family protein & Signal & secreted (5) & NU & EX \\
\hline Bm1_01300 & Alpha amylase, catalytic domain containing protein & Signal & unknown (1) & $\mathrm{CY}$ & EX \\
\hline Bm1_25950 & Hypothetical protein & Signal & unknown (2) & ER & EX \\
\hline Bm1_44655 & Fukutin, putative & Signal & unknown (2) & ER & PM \\
\hline Bm1_01585 & Fatty acid elongation protein 3 , putative & Signal & unknown (5) & ER & EX \\
\hline Bm1_56360 & Nematode cuticle collagen $\mathrm{N}$-terminal domain containing protein & Signal & unknown (5) & NU & EX \\
\hline
\end{tabular}

a Values in parentheses from TargetP results represent the reliability class (RC), from 1 to 5 , where 1 indicates the strongest prediction. For PSORTII, location with the highest percentage score is shown. For ProtComp, the integral prediction of protein location is shown. CY, cytoplasm; ER, endoplasmic reticulum; EX, extracellular; GO, Golgi apparatus; NU, nuclear; PE, peroxisome; PM, plasma membrane; MT, mitochondria. 
A

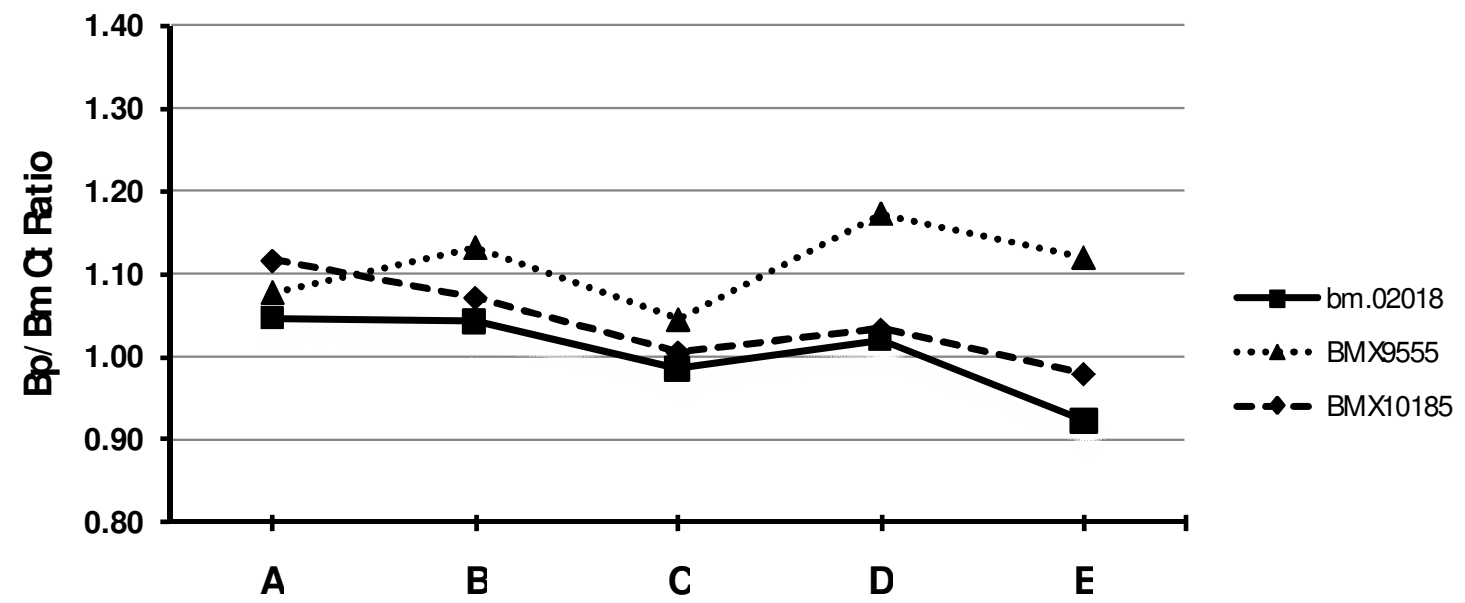

Biological Samples

B

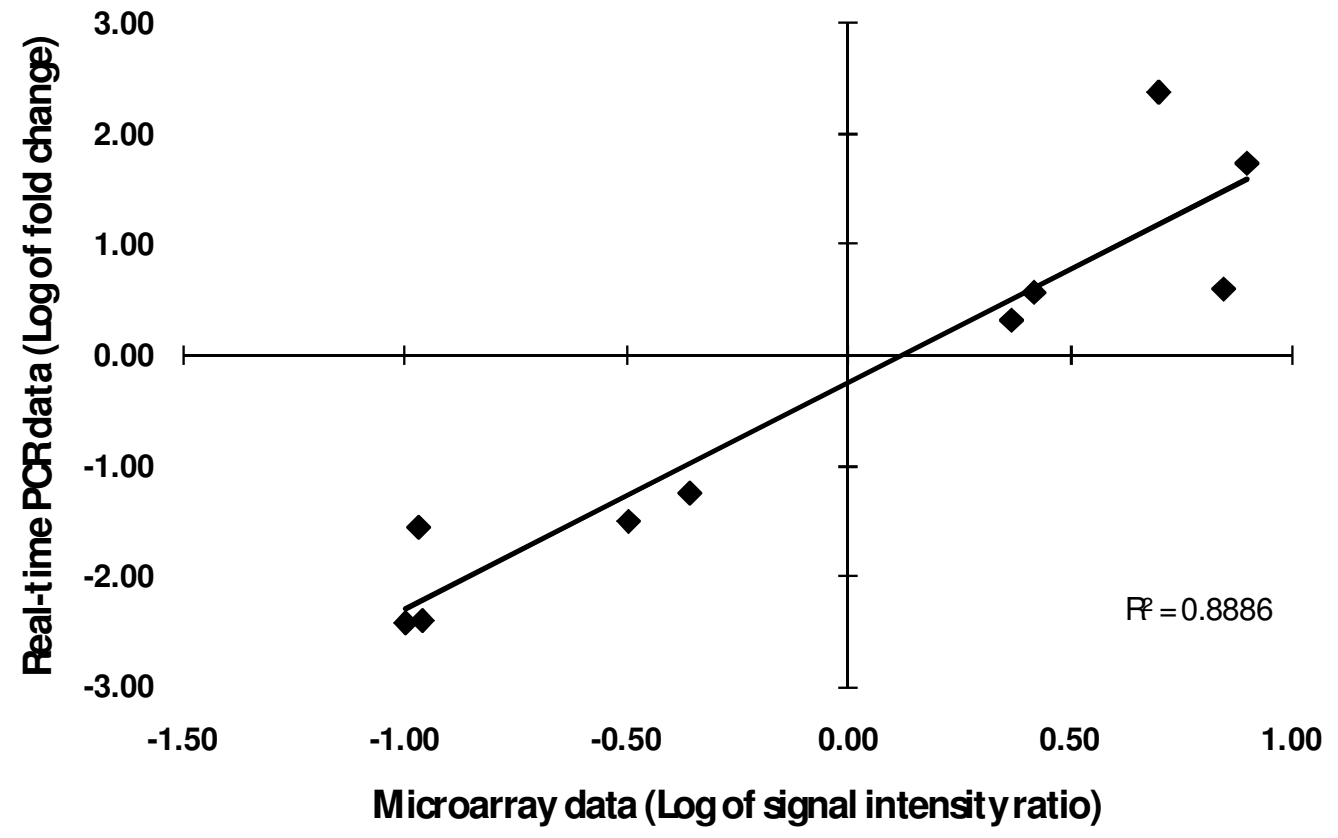

Figure 3 Comparison of real-time PCR and microarray data of ten randomly selected genes. Real-time PCR analysis was performed on CDNA derived from five biological samples of B. pahangi and B. malayi microfilariae with similar age distribution. (A) The ratio of Ct values obtained following real-time PCR analysis using primers flanking three probes shown to have similar signal intensities in the microarray data. Probe bm.02018, which represented Pub locus Bm1_34045, was assigned as the endogenous control gene. (B) Comparison of log-transformed microarray data (three biological samples) and real-time PCR data (five biological samples) of the ten representative genes showing good agreement of expression values obtained from both methodologies. Biological samples used for real-time PCR analysis were independent of those used for microarray analysis. 
Table 5: Sequence of gene-specific primers used for real-time PCR analysis.

\begin{tabular}{|c|c|c|}
\hline Pub locus & Forward primer & Reverse primer \\
\hline Bm1_49585 & GACCGAAACAAGAAAGGAAGCCGA & ACTTTCATCATCATTATGTCGTCGCG \\
\hline Bm1_11050 & GATGAAACGTTTCATGAGATG & GATAATCCTCCAAAACTACCG \\
\hline Bm1_02070 & AATAATTGGATTTCGAGTGAGAC & TTCATGCATAATTACCAGTTTCG \\
\hline Bm1_28420 & AAGGCTTCGATATGTTTCCATC & CTAACCACTAGTACATATTGTCT \\
\hline Bm1_42865 & AAACATGCAGTTAATGCACGAG & TGCTTGCAATTCATTCCTCACT \\
\hline Bm1_19100 & GCCACAAGGTATGCAACCACA & GTGTCTGCGTGGGTGGTAAC \\
\hline Bm1_25950a & GCTAACTTCAATGCACTTAGTG & AAATAAGAAACAACGTTTGATACAG \\
\hline Bm1_01300a & CAATTTTCAGACTCAATCTACTG & AGGTAAGTATTTGTCAACGTTTG \\
\hline Bm1_44655a & GTACGAAAGCAAGGATTCTGCT & CAGAATACTTTGTAGGTATCATTC \\
\hline Bm1_50670a & GTATTAGCTCAAGCAATGGAAG & СТААСТАСТАТСАТСАСТАТТАТС \\
\hline bm.02018 & AGAATCAAATGTCCAGGAAG & CTGTGAGAGGAAGATAGTC \\
\hline BMX9555b & TTGATGTAGATGCTTTAACAAGTGCTGCTC & AAGAAATTGTCGACAAAGTCCGCAAG \\
\hline BMX10185b & CCGCACAACAATCCTCACTTGCT & TGTGTTACTGCTATTAACATCCTCACTGCC \\
\hline
\end{tabular}

Each primer is written in the 5 ' to 3 ' direction.

a Indicates the genes whose probe regions were amplified and sequenced using the primers shown.

b Indicates the probes whose primers were used to determine a suitable endogenous control for real-time PCR analysis.

with respect to this study, we intentionally selected five probes on the BmV2 chip that had a B. malayi to B. pahangi signal intensity ratio between three to ten and were able to show that the sequence of these oligonucleotide probes matched the cognate regions on both the $B$. malayi and $B$. pahangi genes. In addition, the microarray data as a whole did not show any bias towards $B$. malayi in the total number of preferentially up-regulated gene transcripts. Indeed, there were slightly more gene transcripts that were up-regulated in B. pahangi than in $B$. malayi microfilariae. These findings taken together suggest that B. pahangi and B. malayi may have almost identical gene sequences to the extent that it would be possible to obtain biologically meaningful gene transcription profiles of $B$. malayi and B. pahangi microfilariae using the $\mathrm{BmV} 2$ array with minimal bias toward $B$. malayi.

That said, it is possible that there are B. pahangi genes whose nucleotide sequence in the coding region differs slightly from that of their orthologs in $B$. malayi, hence introducing probe-bias towards $B$. malayi transcripts in a cross-species hybridization microarray analysis. For this reason, this study focused mainly on genes whose transcripts appeared to be preferentially up-regulated in $B$. pahangi microfilariae; a conservative approach bearing in mind that genes unique to $B$. pahangi and those with extensive mismatch between the probe and target cDNA sequence may not have been detected in the microarray analysis.

The genes that were classified as up-regulated in either species were manually mapped into the KEGG pathway groups. The composition of genes in some of the KEGG pathway groupings of up-regulated genes in $B$. pahangi and $B$. malayi microfilariae revealed some noteworthy differences. For instance in B. malayi microfilariae, transcripts coding for proteins containing a zinc finger domain accounted for 24 of the 34 (70\%) transcripts that code for proteins involved in the processing of genetic information compared to only 2 out of 24 (8\%) in B. pahangi microfilariae. The zinc finger domain-containing proteins are thought to be mainly involved with the regulation of transcription [28]. The reason for the bias in $B$. malayi towards having higher numbers of up-regulated transcripts of zinc finger domain-containing proteins is at present not clear.

Melanotic encapsulation is a major antimicrobial innate immune defense mechanism in insects that involves the rapid deposition of melanin around invading microbes [9]. Molecules on the surface of invading pathogens are thought to be one of the triggers of melanin synthesis in insects. The proteins and molecules on the surfaces of $B$. malayi and B. pahangi microfilariae have been shown to be similar with only slight differences in the molecular weights of some proteins [10]. Microfilarial proteins likely to modulate the vertebrate and mosquito immune response are those that are likely to be secreted by or on the surface of the microfilariae. Our microarray data showed that both species had, between them, a total of sixty-six preferentially expressed transcripts, twenty seven in $B$. pahangi and thirty nine in $B$. malayi, that coded for proteins likely to be secreted by or on the surface of the microfilariae. As microfilariae are exposed to 
both vertebrate and mosquito immune responses in quick succession, it is possible that some of these proteins may have a dual function in helping to promote microfilaria survival in midst of a human or mosquito immune response. Among transcripts preferentially transcribed in B. pahangi microfilariae and identifiable as coding for known immunomodulatory proteins were a putative homolog of the transforming growth factor-beta (TGFbeta) [29], three putative serpins [30] and two FKBP-type peptidyl-prolyl cis-trans isomerase proteins [31,32].

Human TGF-beta is a ubiquitous protein that plays a role in most biological processes. With regard to immunity, TGF-beta acts as an inhibitory cytokine that has an important role, together with interleukin-10, in down regulating immunity and hence maintain self-tolerance and immune homeostasis $[29,33]$. The B. malayi ortholog of TGF-beta, designated as Bm-TGH-2, is expressed mainly in mature adult parasites and the microfilariae; two of the nematode's life stages that are constantly exposed to the human immune response factors [34]. It is, therefore, plausible that the $\mathrm{Bm}-\mathrm{TGH}-2$ gene in $B$. pahangi may mimic the functions of human TGF-beta by modulating the vertebrate immune response, thereby promoting parasite survival within the vertebrate host [34].

In melanoma cells, TGF-beta has been shown to significantly inhibit melanin synthesis by increasing the rate of degradation of tyrosinase, a rate-limiting enzyme in the melanogenesis pathway [35,36]. In the Brugia microfilariae-Ar. subalbatus interaction, most B. pahangi microfilariae are not melanized whereas the $B$. malayi microfilariae are melanized and destroyed in the mosquito hemocoel [7], suggesting that the B. pahangi microfilariae actively subvert or are deficient of the elements that trigger the synthesis of melanin in Ar. subalbatus. In light of our microarray data showing that B. pahangi microfilariae had significantly higher transcript levels of the TGF-beta homolog than B. malayi microfilariae, it is possible that $B$. pahangi-derived TGF-beta may negatively modulate the synthesis of melanin, essential in the melanization immune response in Ar. subalbatus, by a similar mechanism as that shown to occur in melanoma cells.

Serpins are proteins that inhibit the serine proteases that control proteolytic cascades in various cellular processes. In vertebrates, serpins function to mitigate the damage caused by proteases involved in the body's immune response to injury such as inflammation and coagulation [30,37]. In the case of insects, serpins have been shown to negatively regulate the proteolytic cascade of serine proteases that activate phenoloxidase, a key enzyme in the biochemical synthesis of melanin $[38,39]$. The effect, if any, that microfilaria-derived serpins have on the vertebrate immune system remains unclear
[40,41]. In the case of mosquito-parasite interactions, RNAi studies have shown that mosquito-derived serpins influence the outcome of malaria parasite infections in a parasite species-specific manner whereby the knockdown of Anopheles gambiae serpin 2 (SRPN2) leads to markedly reduced numbers of $P$. berghei oocysts in the A. gambiae midgut [42] but has no effect on the development of P. falciparum oocysts [43]. To date, there have been no reported functional studies on the effect of Brugia microfilaria serpins on the mosquito melanotic immune response. According to the recently published draft of the B. malayi genome [44], there are thirteen serpin locus tags implying that there may be up to thirteen possible serpin genes in B. malayi, however only 8 were represented on the BmV2 array. The microarray results in this study showed that B. pahangi microfilariae had two to five times higher transcript levels of three putative serpins than $\operatorname{did} B$. malayi microfilariae. It may be that these $B$. pahangi-derived serpins modulate the melanization immune response in Ar. subalbatus.

\section{Conclusion}

In nature, the microfilariae of both species encounter the vertebrate and insect immune responses in fairly quick succession following their ingestion in a blood meal by a mosquito. In order for the microfilariae to survive in both the vertebrate and mosquito hosts, it is conceivable that the microfilariae possess a mechanism that functions to modulate both the vertebrate and mosquito immune systems. In the current study, we identified transcripts that are preferentially expressed in B. pahangi and B. malayi microfilariae. These comparative transcriptome data will be of interest to researchers keen on understanding the intrinsic difference, at the molecular level, between $B$. malayi and $B$. pahangi microfilariae especially because these microfilariae elicit such different immune response outcomes in the mosquito, Ar. subalbatus. In addition, we further identified transcripts that encode for proteins that are predicted to be secreted or located on the surface of microfilariae and which could have a significant and dual role in the successive interaction of the microfilaria with the vertebrate and mosquito hosts. Consequently, some of these proteins may serve as ideal targets for intervention strategies against the transmission of Brugian lymphatic filariasis.

\section{Methods}

\section{Parasite material}

B. pahangi and sub-periodic B. malayi microfilariae of similar age distribution, harvested from the intra-peritoneal cavity of experimentally infected jirds, were provided by the NIH/NIAID Filariasis Research Reagent Repository Center (FR3), Parasite Resources Division, College of Veterinary Medicine, University of Georgia, Athens, GA. 
The FR3 protocol for the experimental infection of jirds with B. malayi and B. pahangi and the subsequent collection of microfilariae was as follows. Approximately 1000 each of infective third-stage larvae (L3) of B. malayi and B. pahangi, collected from infected Ae. aegypti, were injected on the same day or not more than a week apart into the peritoneal cavity of recipient jirds. The microfilariae were recovered from the peritoneal cavity of recipient jirds by peritoneal washing using RPMI 1640 medium. On each occasion, the B. malayi microfilariae were recovered from the peritoneal cavity of a single euthanized jird as were $B$. pahangi microfilariae. The microfilariae recovered from the peritoneal cavity of infected jirds were transferred to a $50 \mathrm{ml}$ centrifuge tube and washed five times with Hanks' Buffered Salt Solution (HBSS). An aliquot of the microfilariae was removed and examined using a microscope to ascertain microfilaria viability and number.

\section{Microarray slide}

The B. malayi Version 2 oligonucleotide array (BmV2) was obtained from the NIH/NIAID FR3, Molecular Resources Division, Clark Science Center, Smith College, Northampton, MA. The BmV2 array was comprised of 18104 probes (65 mer) derived from The Institute for Genomic Research (TIGR) databases. There were 15412 probes derived from the extensive TIGR $B$. malayi gene indices, genomic gene models and EST database, 1016 probes derived from the TIGR Onchocerca volvulus gene indices, 872 probes from the TIGR W. bancrofti gene indices and EST clusters, and 804 probes from the genome of the Wolbachia endosymbiont.

\section{RNA preparation for microarray analysis}

Total RNA was isolated from three independent biological samples of $B$. malayi and $B$. pahangi microfilariae using TRIzol reagent (Invitrogen, CA) as per the manufacturer's instructions with minor modifications to the homogenization step. Briefly, approximately two to three million microfilariae of each species were re-suspended in TRIzol reagent, a $3.0 \mathrm{~mm}$ stainless-steel bead (Retsch Inc., PA) was added, and the suspension was vortexed at maximum speed for $30 \mathrm{~min}$ at $4^{\circ} \mathrm{C}$. The steel ball was removed and the suspension homogenized four times for 30 s each using a rotor-stator (PowerGen 125; Fisher Scientific, PA) fitted with a soft tissue probe (OmniTips, GA) and set at two thirds to three quarters speed. A final homogenization step was at three quarters speed for 1 min. The suspension was centrifuged at $12000 \mathrm{~g}$ for 10 $\min$ at $4^{\circ} \mathrm{C}$. Total RNA was extracted from the supernatant following the TRIzol protocol and the concentration and purity was estimated spectrophotometrically. The total RNA quality was determined using an Agilent 2100 bioanalyzer (Agilent Technologies, CA).

\section{First-strand synthesis and microarray hybridization}

The first-strand synthesis and microarray hybridizations were carried out at the Washington University Genome Sequencing Center, St Louis, MO. Equal amounts of cDNA prepared from total RNA isolated from threepaired $B$. malayi and B. pahangi microfilariae samples were competitively hybridized under stringent conditions on the BmV2 array. For RNA expression level comparison, B. malayi and B. pahangi fluorophore-specific cDNA samples were paired. First strand cDNA was generated by oligo-dT primed reverse transcription (Superscript II; Invitrogen) utilizing the 3DNA Array 900 kit (Genisphere, PA). Briefly, the fluorophore specific oligo$\mathrm{dT}$ primer was added to $2 \mu \mathrm{g}$ of total RNA and the solution incubated at $80^{\circ} \mathrm{C}$ for 5 min then cooled on ice for 2 min. cDNA was synthesized according to standard protocols. Each biological sample pair was re-suspended in a formamide-based hybridization buffer and Array 50dT blocker (Genisphere, PA). Two hybridizations were carried out in a sequential manner. The primary hybridization was performed by adding the cDNA samples of both microfilariae species to the microarray slide under a supported glass coverslip (Erie Scientific, $\mathrm{NH}$ ) at $43^{\circ} \mathrm{C}$ for 16 $20 \mathrm{~h}$ at high humidity. Prior to the secondary hybridization, the slide was gently submerged in $2 \times$ SSC, $0.2 \%$ SDS at $43^{\circ} \mathrm{C}$ for $11 \mathrm{~min}$, transferred to $2 \times \mathrm{SSC}$ at RT for $11 \mathrm{~min}$ followed by an incubation in $0.2 \times \mathrm{SSC}$ at RT for $11 \mathrm{~min}$ and then spun dry by centrifugation. The secondary hybridization was carried out using the complimentary capture reagents provided in the 3DNA Array 900 kit (Genisphere, PA). Both the 3DNA capture reagents with Cy3 and Cy5, in a SDS-based hybridization buffer, were added to the microarray slide under a supported glass coverslip. The slide was incubated at $65^{\circ} \mathrm{C}$ for $4 \mathrm{~h}$ at high humidity in the dark. At hybridization termination, the slide was washed as described above. A dye-swap was carried out in order to alleviate concerns about gene-specific dye bias. There were three technical replicates for each of the three biological samples, resulting in a total of nine DNA microarray slides used in this study.

\section{Data acquisition}

The slides were scanned on a Perkin Elmer ScanArray Express HT scanner (Perkin-Elmer, MA) to detect Cy3 and Cy5 fluorescence at 543 and $633 \mathrm{~nm}$, respectively. Laser power was kept constant for $\mathrm{Cy} 3 / \mathrm{Cy} 5$ scans for all slides. The gridding and analysis of images were performed using ScanArray v3.0 (Perkin-Elmer, MA).

\section{Statistical analysis of microarray data}

Fluorescence intensities obtained for Cy3 by $\mathrm{Cy} 5$ for each array were converted into logarithm base 2 values and plotted. The images were assessed for plausibility, and deviations from the expected point cloud were noted. 
The microarray data was statistically modeled using a Linear Mixed Model to account for the array hybridization and dye variability $[45,46]$. The residuals from this model were then modeled for nematode species within each probe using a another Linear Mixed Model. An Ftest for species difference was then computed. The p-values for differences were then sorted into ascending order and cutoff values were established for $\mathrm{p}$-values using a Benjamini and Hochberg false discovery rate (FDR) of $0.05[47,48]$. The FDR is a statistically stringent manipulation that compensates for multiple hypothesis testing in data involving multiple comparisons.

\section{RNA preparation and real-time PCR analysis}

Total RNA was isolated from five-paired B. malay $i$ and $B$. pahangi microfilariae samples using the RNeasy kit (Qiagen, CA) and the contaminating genomic DNA in the total RNA was removed using the TURBO DNA-free ${ }^{\mathrm{TM}}$ kit (Ambion, TX). The first strand cDNA was generated using the SuperScript III First-Strand Synthesis System for RT-PCR (Invitrogen, CA). Thirteen Brugia genes in the microarray data were selected for validation using real-time PCR. The sequences of the selected $B$. malayi genes were retrieved from GenBank and gene-specific primers, which would amplify 200 to 400 bp fragments, were designed. The PCRs were set-up using Power SYBR Green PCR Master Mix (Applied Biosystems, CA) on 96well plates in $20 \mu \mathrm{l}$ reaction volumes with equal amounts of cDNA derived from $B$. malay $i$ and B. pahangi microfilariae as templates. The real-time PCR was carried out on an Applied Biosystems 7300 Real-Time PCR System (Applied Biosystems, CA) and the cycle threshold values $(\mathrm{Ct})$ obtained were used to determine the relative quantity of each target gene between the two Brugia species using the comparative $\mathrm{Ct}$ method [25]. Five biological samples were analyzed independently with each selected gene run in triplicate.

\section{Functional classification of clusters up-regulated in Brugia microfilariae}

For this study, the probes with FDR estimates of 5\% and signal intensity ratios of 2 or higher, with respect to either species, were considered preferentially expressed and prompted further analysis. In the first analysis, these preferentially expressed genes were manually grouped based how they mapped into the Kyoto Encyclopedia of Genes and Genomes (KEGG) biological pathway [49]. Additional mapping of some genes on the BmV2 array into the KEGG pathway was done based on their annotations on the BmV2 array. In the second analysis, the preferentially expressed genes that encoded proteins with a signal sequence, as predicted by the SignalP algorithm [50], were further grouped according to the suggested localization of the proteins they encode as predicted by the protein structure and localization algorithms TargetP [50] and PSORTII [24] hosted by the ExPASy proteomics server of the Swiss Institute of Bioinformatics, and the on-line protein localization algorithm, ProtComp, from Softberry Inc http://www.softberry.com/.

\section{Sequence comparison between B. pahangi and B. malayi genes}

Twenty-two nucleotide sequences of B. pahangi genes, complete or partial, deposited in GenBank were selected and their nucleotide sequences compared with their orthologs in B. malayi using the NCBI's BLAST program. The output of the BLAST analysis was recorded. To determine whether the nucleotide sequences of the probes on the BmV2 array were identical to their cognate regions in the $B$. malayi and $B$. pahangi genes, PCRs were carried out using primers that flanked the probe sequence with $B$. malayi and B. pahangi genomic DNA as templates. The PCR products were purified (QIAquick PCR Purification Kit, Qiagen, CA) and sequenced from both ends using the PCR primers. The nucleotide sequences of the PCR products of both species were aligned with the probe sequence. Sequence alignment was done using the VectorNTI software (Invitrogen, CA).

\section{Additional material}

\begin{abstract}
Additional file 1 Brugia gene transcripts that are up-regulated in $B$. pahangi microfilariae. This file contains a list of genes represented by probes on the microarray that had signal intensity ratios of 2 or higher in $B$. pahangi microfilariae and a FDR estimate of $5 \%$. These genes were considered preferentially expressed and manually mapped into the appropriate KEGG pathway. a Genes represented by more than one probe on the BmV2 array. For these genes, an average of the $p$-value and signal intensity ratio is shown. Genes in each of the KEGG pathway groups are ranked according to their ratios.
\end{abstract}

Additional file $\mathbf{2}$ Brugia gene transcripts that are up-regulated in $B$. malayi microfilariae. This file contains a list of genes represented by probes on the microarray that had signal intensity ratios of 2 or higher in $B$. malayi microfilariae and a FDR estimate of $5 \%$. These genes were considered preferentially expressed and manually mapped into the appropriate KEGG pathway. ${ }^{a}$ Genes represented by more than one probe on the BmV2 array. For these genes, an average of the $p$-value and signal intensity ratio is shown. Genes in each of the KEGG pathway groups are ranked according to their ratios.

\section{Authors' contributions}

MMK prepared the RNA samples used for real-time PCR analysis and performed the real-time PCR analysis, sequence alignments, analysis of the microarray data, generation of the gene lists, and drafting of the manuscript. LBH performed the statistical analysis of the microarray data. BTB designed the study, prepared the RNA samples used in the microarray analysis, supervised all aspects of data collection and analysis, and revised the manuscript. All authors read and approved the final manuscript.

\section{Acknowledgements}

We thank the NIH/NAID Filariasis Research Reagent Repository Center, which provided the Brugia microfilariae and the BmV2 array used in this study, and Dr. Elodie Ghedin for providing an annotated gene list for the array. The Washing ton University Genome Sequencing Center performed the microarray hybridization. This study was partially funded by a University of Missouri System Research Board grant. 


\section{Author Details}

'Department of Veterinary Pathobiology, College of Veterinary Medicine, University of Missouri, Columbia, MO 65211, USA and 2Department of Statistics, College of Arts and Science, University of Missouri, Columbia, MO 65211, USA

Received: 27 July 2009 Accepted: 7 April 2010

Published: 7 April 2010

\section{References}

1. Mak JW: Epidemiology of lymphatic filariasis. Ciba Found Symp 1987 127:5-14.

2. McReynolds LA, DeSimone SM, Williams SA: Cloning and comparison of repeated DNA sequences from the human filarial parasite Brugia malayi and the animal parasite Brugia pahangi. Proc Natl Acad Sci USA 1986, 83(3):797-801.

3. McReynolds LA, Poole C, Hong Y, Williams SA, Partono F, Bradley J: Recent advances in the application of molecular biology in filariasis. Southeast Asian J Trop Med Public Health 1993, 24(Suppl 2):55-63.

4. Xie H, Bain O, Williams SA: Molecular phylogenetic studies on Brugia filariae using Hha I repeat sequences. Parasite 1994, 1(3):255-260.

5. Nayar JK, Knight JW, Vickery AC: Susceptibility of Anopheles quadrimaculatus (Diptera: Culicidae) to subperiodic Brugia malayi and Brugia pahangi (Nematoda: Filarioidea) adapted to nude mice and jirds. JMed Entomol 1990, 27(3):409-411.

6. Kobayashi MON, Tsuruoka H, Chigusa Y, Mishima S: Studies on filariasis VII: Histological observation on the encapsulated Brugia malayi larvae in the abdominal haemocoel of the mosquitoes, Armigeres subalbatus. Jpn J Sanit Zool 1986, 37:59-65.

7. Beerntsen BT, Luckhart S, Christensen BM: Brugia malayi and Brugia pahangi: inherent difference in immune activation in the mosquitoes Armigeres subalbatus and Aedes aegypti. J Parasitol 1989, 75(1):76-81.

8. Zhao X, Ferdig MT, Li J, Christensen BM: Biochemical pathway of melanotic encapsulation of Brugia malayi in the mosquito, Armigeres subalbatus. Dev Comp Immunol 1995, 19(3):205-215.

9. Christensen BM, Li J, Chen CC, Nappi AJ: Melanization immune responses in mosquito vectors. Trends Parasitol 2005, 21(4):192-199.

10. Maizels RM, Partono F, Oemijati S, Denham DA, Ogilvie BM: Cross-reactive surface antigens on three stages of Brugia malayi, B. pahangi and $B$. timori. Parasitology 1983, 87(Pt 2):249-263.

11. Araujo AC, Souto-Padron T, de Souza W: Cytochemical localization of carbohydrate residues in microfilariae of Wuchereria bancrofti and Brugia malayi. J Histochem Cytochem 1993, 41(4):571-578.

12. Klonisch T, Bardehle G, Linder D, Boschek B, Schott HH, Zahner H, Stirm S: The sheaths of Brugia microfilariae: isolation and composition. Parasitol Res 1991, 77(5):448-451.

13. Fuhrman JA, Urioste SS, Hamill B, Spielman A, Piessens WF: Functional and antigenic maturation of Brugia malayi microfilariae. Am J Trop Med Hyg 1987, 36(1):70-74.

14. Wang X, Fuchs JF, Infanger LC, Rocheleau TA, Hillyer JF, Chen CC, Christensen BM: Mosquito innate immunity: involvement of beta 1,3glucan recognition protein in melanotic encapsulation immune responses in Armigeres subalbatus. Mol Biochem Parasitol 2005 139(1):65-73.

15. Stoughton RB: Applications of DNA microarrays in biology. Annu Rev Biochem 2005, 74:53-82.

16. DeRisi J, Penland L, Brown PO, Bittner ML, Meltzer PS, Ray M, Chen Y, Su YA, Trent JM: Use of a CDNA microarray to analyse gene expression patterns in human cancer. Nat Genet 1996, 14(4):457-460.

17. Bar-Or C, Bar-Eyal M, Gal TZ, Kapulnik Y, Czosnek H, Koltai H: Derivation of species-specific hybridization-like knowledge out of cross-species hybridization results. BMC Genomics 2006, 7:110.

18. Nowrousian M, Ringelberg C, Dunlap JC, Loros JJ, Kuck U: Cross-species microarray hybridization to identify developmentally regulated genes in the filamentous fungus Sordaria macrospora. Mol Genet Genomics 2005, 273(2):137-149.

19. Renn SC, Aubin-Horth N, Hofmann HA: Biologically meaningful expression profiling across species using heterologous hybridization to a CDNA microarray. BMC Genomics 2004, 5(1):42.

20. Breathnach R, Benoist C, O'Hare K, Gannon F, Chambon P: Ovalbumin gene: evidence for a leader sequence in mRNA and DNA sequences at the exon-intron boundaries. Proc Natl Acad Sci USA 1978 75(10):4853-4857.

21. Ghedin E, Wang S, Spiro D, Caler E, Zhao Q, Crabtree J, Allen JE, Delcher AL, Guiliano DB, Miranda-Saavedra D, et al.: Draft genome of the filarial nematode parasite Brugia malayi. Science 2007, 317(5845):1756-1760.

22. Nielsen $\mathrm{H}$, Engelbrecht J, Brunak S, von Heijne G: Identification of prokaryotic and eukaryotic signal peptides and prediction of their cleavage sites. Protein Eng 1997, 10(1):1-6

23. Emanuelsson $\mathrm{O}$, Nielsen $\mathrm{H}$, Brunak S, von Heijne G: Predicting subcellular localization of proteins based on their $\mathrm{N}$-terminal amino acid sequence. J Mol Biol 2000, 300(4):1005-1016.

24. Nakai K, Horton P: PSORT: a program for detecting sorting signals in proteins and predicting their subcellular localization. Trends Biochem Sci 1999, 24(1):34-36.

25. Livak KJ, Schmittgen TD: Analysis of relative gene expression data using real-time quantitative PCR and the 2(-Delta Delta $C(T))$ Method. Methods 2001, 25(4):402-408.

26. Bar-Or C, Czosnek H, Koltai H: Cross-species microarray hybridizations: a developing tool for studying species diversity. Trends Genet 2007 23(4):200-207.

27. Buckley BA: Comparative environmental genomics in non-model species: using heterologous hybridization to DNA-based microarrays. J Exp Biol 2007, 210(Pt 9):1602-1606.

28. Kang JS, Kim JS: Zinc finger proteins as designer transcription factors. J Biol Chem 2000, 275(12):8742-8748.

29. Taylor AW: Review of the activation of TGF-beta in immunity. J Leukoc Biol 2009, 85(1):29-33.

30. Mangan MS, Kaiserman D, Bird PI: The role of serpins in vertebrate immunity. Tissue Antigens 2008, 72(1):1-10.

31. Kang CB, Hong Y, Dhe-Paganon S, Yoon HS: FKBP family proteins: immunophilins with versatile biological functions. Neurosignals 2008, 16(4):318-325

32. Ma D, Carlow CK: Molecular characterization of FKBP13 from filarial parasites. Mol Biochem Parasitol 1999, 99(2):263-267.

33. Wan YY, Flavell RA: TGF-beta and regulatory $T$ cell in immunity and autoimmunity. J Clin Immunol 2008, 28(6):647-659.

34. Gomez-Escobar N, Gregory WF, Maizels RM: Identification of tgh-2, a filarial nematode homolog of Caenorhabditis elegans daf-7 and human transforming growth factor beta, expressed in microfilarial and adult stages of Brugia malayi. Infect Immun 2000, 68(11):6402-6410.

35. Kim DS, Park SH, Park KC: Transforming growth factor-beta1 decreases melanin synthesis via delayed extracellular signal-regulated kinase activation. Int J Biochem Cell Biol 2004, 36(8):1482-1491.

36. Martinez-Esparza M, Jimenez-Cervantes C, Beermann F, Aparicio $P$, Lozano JA, Garcia-Borron JC: Transforming growth factor-beta1 inhibits basal melanogenesis in B16/F10 mouse melanoma cells by increasing the rate of degradation of tyrosinase and tyrosinase-related protein-1. J Biol Chem 1997, 272(7):3967-3972.

37. van Gent D, Sharp P, Morgan K, Kalsheker N: Serpins: structure, function and molecular evolution. Int J Biochem Cell Biol 2003, 35(11):1536-1547.

38. Cerenius L, Soderhall K: The prophenoloxidase-activating system in invertebrates. Immunol Rev 2004, 198:116-126.

39. Nappi AJ, Frey F, Carton Y: Drosophila serpin 27A is a likely target for immune suppression of the blood cell-mediated melanotic encapsulation response. J Insect Physiol 2005, 51(2):197-205

40. Stanley P, Stein PE: BmSPN2, a serpin secreted by the filarial nematode Brugia malayi, does not inhibit human neutrophil proteinases but plays a noninhibitory role. Biochemistry 2003, 42(20):6241-6248.

41. Zang X, Yazdanbakhsh M, Jiang H, Kanost MR, Maizels RM: A novel serpin expressed by blood-borne microfilariae of the parasitic nematode Brugia malayi inhibits human neutrophil serine proteinases. Blood 1999, 94(4):1418-1428.

42. Michel K, Budd A, Pinto S, Gibson TJ, Kafatos FC: Anopheles gambiae SRPN2 facilitates midgut invasion by the malaria parasite Plasmodium berghei. EMBO Rep 2005, 6(9):891-897.

43. Michel K, Suwanchaichinda C, Morlais I, Lambrechts L, Cohuet A, AwonoAmbene PH, Simard F, Fontenille D, Kanost MR, Kafatos FC: Increased melanizing activity in Anopheles gambiae does not affect development of Plasmodium falciparum. Proc Natl Acad Sci USA 2006, 103(45):16858-16863.

44. Ghedin E, Wang S, Spiro D, Caler E, Zhao Q, Crabtree J, Allen JE, Delcher AL, Guiliano DB, Miranda-Saavedra D, Angiuoli SV, Creasy T, Amedeo P, Haas 
B, El-Sayed NM, Wortman JR, Feldblyum T, Tallon L, Schatz M, Shumway M, Koo H, Salzberg SL, Schobel S, Pertea M, Pop M, White O, Barton GJ,

Carlow CK, Crawford MJ, Daub J, Dimmic MW, Estes CF, Foster JM, Ganatra M, Gregory WF, Johnson NM, Jin J, Komuniecki R, Korf I, Kumar S, Laney S, Li BW, Li W, Lindblom TH, Lustigman S, Ma D, Maina CV, Martin DM,

McCarter JP, McReynolds L, Mitreva M, Nutman TB, Parkinson J, PeregrínAlvarez JM, Poole C, Ren Q, Saunders L, Sluder AE, Smith K, Stanke M, Unnasch TR, Ware J, Wei AD, Weil G, Williams DJ, Zhang Y, Williams SA, Fraser-Liggett C, Slatko B, Blaxter ML, Scott AL: Draft genome of the filarial nematode parasite Brugia malayi. Science 2007, 317(5845):1756-1760

45. Wolfinger RD, Gibson G, Wolfinger ED, Bennett L, Hamadeh H, Bushel P, Afshari C, Paules RS: Assessing gene significance from CDNA microarray expression data via mixed models. J Comput Bio/ 2001, 8(6):625-637.

46. Kerr MK: Linear models for microarray data analysis: hidden similarities and differences. J Comput Biol 2003, 10(6):891-901.

47. Benjamini Y, Drai D, Elmer G, Kafkafi N, Golani I: Controlling the false discovery rate in behavior genetics research. Behav Brain Res 2001, 125(1-2):279-284

48. Storey JD, Tibshirani R: Statistical significance for genomewide studies. Proc Natl Acad Sci USA 2003, 100(16):9440-9445.

49. Kanehisa M, Goto S: KEGG: kyoto encyclopedia of genes and genomes. Nucleic Acids Res 2000, 28(1):27-30.

50. Emanuelsson O, Brunak S, von Heijne G, Nielsen H: Locating proteins in the cell using TargetP, SignalP and related tools. Nat Protoc 2007, 2(4):953-971.

doi: 10.1186/1471-2164-11-225

Cite this article as: Kariuki et al., Differential transcript expression between the microfilariae of the filarial nematodes, Brugia malayi and B. pahangi $B M C$ Genomics 2010, 11:225

Submit your next manuscript to BioMed Central and take full advantage of:

- Convenient online submission

- Thorough peer review

- No space constraints or color figure charges

- Immediate publication on acceptance

- Inclusion in PubMed, CAS, Scopus and Google Scholar

- Research which is freely available for redistribution

Submit your manuscript at www.biomedcentral.com/submit
Ciomed Central 